\title{
Contagion of network products in small-world networks
}

\author{
Hüseyin Iikizler ${ }^{1,2}$ \\ Received: 16 April 2018 / Accepted: 10 May 2019 / Published online: 20 May 2019 \\ (c) Springer-Verlag GmbH Germany, part of Springer Nature 2019
}

\begin{abstract}
We formulate a model in which agents embedded in an exogenous social network decide whether to adopt a new network product or not. In the theoretical part of the paper, we characterize the stochastically stable equilibria for complete networks and cycles. For an arbitrary network structure, we develop a novel graph decomposition method to characterize the set of recurrent communication states, which is a superset of stochastically stable equilibria of the adoption game presented in our model. In the simulation part, we study the contagion process of a network product in small-world networks that systematically represent social networks. We simulate a generalization of the Morris (Rev Econ Stud 67(1):57-78, 2000) Contagion model that can explain the chasm between early adopters and early majority. Our numerical analysis shows that the failure of a new network product is less likely in a highly cliquish network. In addition, the contagion process reaches to steady state faster in random networks than in highly cliquish networks. It turns out that marketers should work with mixed marketing strategies, which will result in a full contagion of a network product and faster contagion rates with a higher probability.
\end{abstract}

Keywords Social network · Contagion · Simulation · Cliquish network · Random network $\cdot$ Small-world network

JEL Classification A14 $\cdot$ C63 $\cdot$ D12 $\cdot$ D71 $\cdot$ D91

\section{Introduction}

The market offers new products every day, but most of them fail to reach mainstream consumers. According to Miller and Muir (2005), communication between consumers often determines the success or failure of a new brand. In many cases, heavy advertising

\footnotetext{
\üseyin İkizler huseyin.ikizler@bilkent.edu.tr

1 Department of Economics, Bilkent University, Ankara, Turkey

2 Presidency of the RT, Presidency of Strategy and Budget, Ankara, Turkey
} 
campaigns are defeated by positive word of mouth. For instance, in 2000, Big Brother and Survivor were rival reality TV programs in England. While there was a massive advertising campaign for Survivor, the producers of Big Brother relied on word of mouth. After a slow start, Big Brother captured the most of audiences. The same is true for in the case of The Blair Witch Project and Godzilla. The success of the Blair Witch Project relative to Godzilla was achieved by internet rumors regarding the content of the film. A different example includes Palm Computing, whose product was able to diffuse throughout the mainstream market by focusing on a specific group of customers. On the contrary, Momenta Corporation tried to reach the mass market with a massive advertising campaign, but they failed to diffuse.

Network product has a value that is contingent or partially dependent on the number of current users of the product (McIntyre and Chintakananda 2014). In our model, an agent gets value from the use of a network product contingent on the number of current users who are known by the agent. We consider a network product as a tool that supports the compatible connections between acquaintances. For instance, a file sharing application is a network product that provides fast and safe sharing between contacts. Agents choose a file sharing application that has a larger number of users known by them. The total number of users of a file sharing application is not that important. As for another motivation of this idea, a film, book, or TV program can be a network product. People want to talk about similar interests with others (Tauber 1972). Gilchrist and Sands (2016) propose that film consumption is partly driven by the preference for shared experience. Besides, agents can imitate others through social networks (Richerson and Boyd 1998; Babutsidze and Cowan 2014). So, to communicate with more friends, agents should allocate their time according to their friends' interests. We formalize this observation in our model by assuming that if more than half of an agent's friends read a particular book or watch a particular TV program, he will probably choose to read that book or watch that TV program. ${ }^{1}$

It is natural to ask when a network product will diffuse with a higher probability in the market. As in the case of Palm Computing, marketers should focus on a specific group of customers at a time. ${ }^{2}$ Besides, there is a chasm between the "early adopters" and "early majority", and crossing this chasm brings the full diffusion of a network product with a higher probability (Moore 2002).

The aim of this paper is twofold. First, we want to analyze what determines the success or failure of a new network product by focusing on the role of network structure. Second, we try to introduce a game theoretical model that can also explain the chasm between early adopters and early majority.

In the theoretical part, we formulate a strategic normal form game in which the payoff of each agent depends on the actions of his neighbors. We study the equilibria of the network product adoption game and how these equilibria change with the given network. We focus on the equilibria that segregate agents into different types of adoption status: adopting (1) or non-adopting (0). Initially, the market offers a new

\footnotetext{
${ }^{1}$ For the sake of simplicity, we assume that the threshold is $1 / 2$ in the theoretical model, but we generalize it in the simulation model.

2 Rogers (2010) partitioned a normal distribution into five consumer groups: innovators (2.5\%), early adopters (13.5\%), early majority (34\%), late majority (34\%) and laggards (16\%).
} 
network product. At each following period, an agent is arbitrarily chosen to consider adopting the new network product. The chosen agent adopts the new network product if more than half of his neighbors adopt the same network product; otherwise, he doesn't change his adoption status. It is always equilibrium when all agents adopt the new network product, so we ignore such equilibria and focus on the structure of those where at least two parties have a different adoption status. We characterize the stochastically stable equilibria for complete networks and cycles. For an arbitrary network structure, we develop a novel graph decomposition method to characterize the set of recurrent communication states, which is a superset of stochastically stable equilibria of the network product adoption game.

In the simulation part, we generalize the Morris (2000) Contagion model. In particular, we consider a set of agents communicating on an exogenously given undirected network. Each agent must choose one of two adoption statuses. For each agent $i$ there exists a threshold probability $p_{i}$ such that adopting a new network product is the best response for an agent if at least $p_{i}$ of his neighbors adopted the same network product. To represent network structures we use Watts and Strogatz's (1998) "small-world" model. This model allows us to analyze two extreme cases: highly cliquish networks and random networks. We calibrate the threshold probabilities for each agent using the parameters in Choi et al. (2010).

The paper proceeds as follows: in Sect. 2, we review the related literature. We propose our theoretical model in Sect. 3. In Sect. 4, we discuss our theoretical results. We introduce our simulation model in Sect. 5. In Sect. 6, we discuss our simulation results. In the last section, we conclude with a discussion of the related literature.

\section{Related literature}

There is vast literature that studies the relation between agents' positions in social network and their behavior (Granovetter 1995; Ioannides and Topa 2010; Sacerdote 2001). However, many of the works take network effects as an aggregate influence by focusing on a total number of adopters (Xiong et al. 2016). Note that the characterization of the equilibria of a diffusion model in a social network depends on the minute details of the network. So, in order to generalize the results, we need heavy theoretical assumptions that may not have economic intuitions (Morris 2000; Lelarge 2012). Recently, however, progress in complexity theory has yielded tools to analyze social network systematically (Boccaletti et al. 2006; Chakravartula 2014).

There are two approaches that are widely used by researchers to simulate agents' adoption decisions (Xiong et al. 2016): the probability-based approach (Bass 1969; Peres 2014) and the threshold-based approach (Granovetter 1978; Morris 2000; Choi et al. 2010; Pegoretti et al. 2012; Lelarge 2012). In the probability-based approach, a non-adopting agent's probability of adopting a new network product depends linearly on external influence and the number of previous adopters. However, there are several drawbacks of a probability-based approach. ${ }^{3}$ For instance, there is no explicit social

\footnotetext{
3 See Kiesling et al. (2012) for a review.
} 
structure and probability-based approaches assume the homogeneity of the potential adopter population (Bass 1969).

We adopt a threshold-based approach because in contrast to the probability based approach it allows agent-based modeling. ${ }^{4}$ As an example, Lelarge (2012) introduces a symmetric percolated threshold model to analyze the diffusion in a large population. In this model, the threshold associated with an agent is a random variable depending on the number of agent's links. Another related model is the Morris (2000) Contagion model, but in contrast to Lelarge (2012), an agent in Morris (2000) adopts a new network product if at least proportion $q$ of his neighbors adopted the same network product. That is, Lelarge (2012) can be seen as a generalization of the Morris (2000) Contagion model.

For the simulation part of the model, a way of analyzing network effect in simulation models is using random networks, but these networks do not share some important properties of social networks (Newman and Watts 1999; Moore and Newman 2000). ${ }^{5}$ For example, in social networks, the probability that three agents are linked together increases if two of them are linked to each other. On the contrary, in a random network, the probability that a link exists between any two agents is uniform. Watts and Strogatz (1998) suggest a "small-world" model which captures this clustering property of social networks. In a small-world network construction algorithm, we start with a regular ring lattice, in which each agent is linked with his nearest $k$ neighbors on either side. ${ }^{6}$ With probability $\beta$, we rewire each link in the network to a random agent. Notice that when $\beta=1$ we have the Erdos and Rényi (1960) model. It shows that, depending on the range of $\beta$ values, networks exhibit properties of both random and regular networks.

In this paper, we assume that agents consider only close friends' decisions on adopting a new network product. On average, each agent will consider 10 of his friends while deciding whether to adopt a new network product or not. This implies that all the simulations are made starting from a regular network of 1000 agents with 10 links each. On the contrary, we confirm Choi et al.'s (2010) results if we start with a perfect 1-lattice in which each agent has precisely 20 links each. From an anthropology perspective, we observe some supporting observations. The social brain hypothesis suggests that ego networks are composed of (Dunbar) layers. The size of the layer increases as emotional closeness decreases (Dunbar 1998; Hill and Dunbar 2003). People give more weight to relationships in inner layers and the magnitude of weight changes quicker in the outer layers (Sutcliffe et al. 2012; Saramäki et al. 2014). These Dunbar layers are also observed in social media and online computer game platforms (Dunbar et al. 2015; Fuchs et al. 2014). Mac Carron et al. (2016) find that the average cumulative layer turns out to hold 4.1, 11.0, 29.8, and 128.9 friends. Hence, we simply assume that agents consider only their friends' adoption status in the first and second layers.

\footnotetext{
4 See Bargigli and Tedeschi (2013) for major trends in agent-based economics.

5 Random networks can be generated in two algorithms. In the first algorithm, we start with any network, then we randomly delete each link with some probability (bond percolation). In the second algorithm, there are $n$ agents and there is a probability of a link $\left(p_{\text {link }}\right)$ between any two agents. A random number is generated for any two agents. If the random number is less than $p_{l i n k}$, then the link between these two agents is formed (Erdos and Rényi's 1960 model).

6 In a regular network each agent has the same number of links.
} 
Our work builds on these prior studies. The key difference is that we build a game theoretical model that analyzes how the network structure affects the speed of diffusion of network product. In our analysis we show that the network product diffuses faster when the network is completely random. We also show that weak cliquishness increases the probability of falling into a trap of under-adoption.

\section{Theoretical model}

\subsection{The network}

There is an exogenously given undirected communication network that connects a finite set of agents $N=\{1, \ldots n\}$. For any network $g$ and for any $i, j \in N,(i, j) \in g$ means that agent $i$ talks to agent $j$. In addition, $N_{i}(g)$ will denote the set of agents linked with agent $i$, formally:

$$
N_{i}(g)=\{j \in N \text { such that }(i, j) \in g\} .
$$

\subsection{The dynamic adoption procedure}

Let's assume that the market offers a new network product. At each period, an agent is arbitrarily chosen to consider adopting the new network product. The chosen agent adopts the new network product if more than half of his neighbors adopt the same network product; otherwise, he doesn't change his adoption status. After the choice is made, with probability $\varepsilon$, it is reversed by a tremble.

These trembles can be thought of as mistakes or exogenous factors (product quality, price, etc.) that influence purchasing transactions (Jackson and Watts 2002b). ${ }^{7}$ Alternatively, the agent who adopts the new network product even though more than half of his neighbors didn't adopt the network product can be interpreted as an "innovator" or "early majority". Likewise, the agent who doesn't adopt the new network product even though more than half of his neighbors did can be interpreted as part of the "late majority" or a "laggard". Disadoption (discontinuance or suspension) of the network product can be considered as the adoption of a competing product. ${ }^{8}$ Again, mistakes or exogenous factors may influence the adoption of a competing product.

For a given network product, the network $g$ is partitioned into two subnetworks: the non-adopting network $\left(g_{0}\right)$ and the adopting network $\left(g_{1}\right)$ such that:

$$
\begin{aligned}
\lim _{t \rightarrow \infty} g_{0}^{t} & =g_{0} \\
\lim _{t \rightarrow \infty} g_{1}^{t} & =g_{1},
\end{aligned}
$$

\footnotetext{
7 We assume that there are no significant differences between competing network products in terms of price and quality; i.e., these differences are assumed to be $\varepsilon$-relevant.

8 See Lehmann and Parker (2017) for a detailed review.
} 
where $g_{0}^{t}$ denotes the subnetwork of the agents who didn't adopt the new network product at the end of the period $t .{ }^{9}$ Respectively, $g_{1}^{t}$ denotes the subnetwork of the agents who adopted the new network product before the end of the period $t$.

Next, we describe the evolutions of the networks $g_{0}$ and $g_{1}$ step by step:

1. At period $t$, an agent $i$ is arbitrarily chosen from the set $N$.

Case 1 If agent $i$ is in the non-adopting network $g_{0}^{t-1}$, i.e. $N_{i}\left(g_{0}^{t-1}\right) \neq \emptyset$.

2. If $2 \times\left|N_{i}\left(g_{0}^{t-1}\right)\right|<\left|N_{i}(g)\right|$, he chooses to adopt the new network product.

3. With probability $\varepsilon$, the choice is reversed by a tremble. That is, with probability $1-\varepsilon, N_{i}\left(g_{0}^{t}\right)=\emptyset$ and $N_{i}\left(g_{1}^{t}\right)=\left\{j \mid j \in N_{i}(g)\right.$ and $\left.N_{j}\left(g_{1}^{t-1}\right) \neq \emptyset\right\}$.

Case 2 If agent $i$ is in the adopting network $g_{1}^{t-1}$, i.e. $N_{i}\left(g_{1}^{t-1}\right) \neq \emptyset$.

$\mathbf{2}^{\prime}$. If $2 \times\left|N_{i}\left(g_{1}^{t-1}\right)\right|<\left|N_{i}(g)\right|$, he chooses to quit using the network product.

$\mathbf{3}^{\prime}$. With probability $\varepsilon$, the choice is reversed by a tremble. That is, with probability $1-\varepsilon, N_{i}\left(g_{1}^{t}\right)=\emptyset$ and $N_{i}\left(g_{0}^{t}\right)=\left\{j \mid j \in N_{i}(g)\right.$ and $\left.N_{j}\left(g_{0}^{t-1}\right) \neq \emptyset\right\}$.

Conditional on the structure of the network $g$, the structures of the networks $g_{0}$ and $g_{1}$ are determined. Precisely, if $(i, j) \in g$ and both agents adopt the network product, then $(i, j) \in g_{1}$. Similarly, if $(i, j) \in g$ and both agents don't adopt the network product, then $(i, j) \in g_{0}$. Note that either $N_{i}\left(g_{0}\right)=\emptyset$ or $N_{i}\left(g_{1}\right)=\emptyset$. While there is no link formation or deletion in the network $g$, the links can be formed or deleted in the subnetworks $g_{0}$ and $g_{1}$. That is, links can only be preserved if the same network product is used. ${ }^{10}$

\subsection{Preliminary definitions}

At time $t$, the state of the system is a vector $s_{t}=\left(s_{t}^{1}, \ldots, s_{t}^{n}\right) \in S \equiv\{0,1\}^{n}$ where $s_{t}^{i}=1$ if agent $i$ adopts the new network product, and $s_{t}^{i}=0$ otherwise. Let $s_{A}$ and $s_{N A}$ denote, respectively, the state where everyone adopts the new network product and the state where no one adopts the new network product.

Let $P$ be a stationary Markov chain defined on a finite state space, $S$, with a set of mutations $P^{\varepsilon}$ where $\varepsilon$ is a scalar parameter that measures the overall level of noise, i.e. $\varepsilon \in(0,1]$.

We assume that the perturbed process can be modeled as a stationary Markov chain on $S$, and the following conditions hold for any $x, y \in S$ :

(i) For any $\varepsilon \in(0,1], P^{\varepsilon}$ is aperiodic and irreducible,

(ii) $P^{\varepsilon}$ converges to $P$ as $\varepsilon$ converges to 0 ,

\footnotetext{
${ }^{9}$ Particularly, the set of nodes of the network $g$ is partitioned into the sets of the nodes of the subnetworks $g_{0}$ and $g_{1}$.

10 The benefit that an agent derives from the use of a network product often depends on the number of his connections who purchase compatible items. For example, PlayStation and Xbox do not offer crossplatform connectivity. So, agents need to have the same video game console to play multiplayer games with their friends.
} 
(iii) $P_{x y}^{\varepsilon}>0$ implies there exists $r(x, y) \geq 0$ such that ${ }^{11}$

$$
0<\lim _{\varepsilon \rightarrow 0} \varepsilon^{-r} \cdot P_{x y}^{\varepsilon}<\infty
$$

These assumptions guarantee that $P^{\varepsilon}$ has a unique stationary distribution $\mu^{\varepsilon}$, for any $\varepsilon>0$. In the end, $\mu^{\varepsilon}$ converges to $\mu$, where $\mu$ is one of the stationary distributions of $P$.

The recurrent communication states of a Markov process $P$ are characterized by:

- For all states in state space $S$, there is a path of zero resistance to some recurrent communication state.

- For any two recurrent communication states $x, y$, the resistance of any path from $x$ to $y$ is positive.

An $s$-tree for a given state $s$ in $S$ is a directed graph such that for every state $s^{\prime}(\neq s)$ there exists a unique directed path leading from $s^{\prime}$ to $s$. The stochastic potential of $\mathbf{s}$ is defined by finding an $s$-tree that minimizes the sum of the resistance of the directed paths.

\subsection{Stochastically stable equilibria}

Let $\mu_{\varepsilon}(\cdot)$ be a limiting distribution of $P(t)$, an adoption process, as $t$ tends to $\infty$. For general dynamic systems this distribution may not exist, but the limit superior of this distribution when $\varepsilon$ converges to 0 always exists, and we denote $\mu=\lim _{\sup _{\varepsilon \rightarrow 0}} \mu_{\varepsilon}$.

Following Foster and Young (1990), $s^{*}$ is stochastically stable if it is in the support of $\mu$, more precisely,

$$
\forall \sigma>0, \quad \mu=\limsup _{\varepsilon \rightarrow 0} \int_{B_{\sigma}\left(s^{*}\right)} \mu_{\varepsilon}(s) d s>0
$$

where $B_{\sigma}\left(s^{*}\right)=\left\{s:\left|s-s^{*}\right|<\sigma\right\}$. In words, $s^{*}$ is stochastically stable if, in the long run, it is almost certain that the system will be in that state as the probability of a tremble tends to zero.

Theorem 1 (Young's Theorem) Let $P$ be a stationary Markov process on a finite state space with a regular perturbation (satisfying (i) to (iii)) of $P, P^{\varepsilon}$, and let $\mu^{\varepsilon}$ be the unique stationary distribution of $P^{\varepsilon}$. Then,

- as $\varepsilon \rightarrow 0, \mu^{\varepsilon} \rightarrow \mu$,

$-s$ is stochastically stable if and only if $s$ is a recurrent communication state that has the minimum stochastic potential.

\footnotetext{
11 Two adjacent states $(x, y)$ differ by at most one agents' adoption decision. Let's say, there is an agent who does not adopt the new network product in the state $x$ and he adopts the new network product in state $y$. Let resistance, $r(x, y)$, be the number of trembles needed to go from $x$ to $y$. A tremble is needed to move from $x$ to $y$ if the agent involved adopts the new network product. Thus, going from $x$ to $y$ takes at most 1 tremble and at least 0 trembles. $r(x, y)=1$ if one tremble is needed to go from $x$ to $y$. The resistance of $a$ path is $r\left(s_{1}, \ldots, s_{k}\right)=\sum_{i=1}^{k-1} r\left(s_{i}, s_{i+1}\right)$.
} 
Using Young's Theorem (1993), we will determine stochastically stable equilibria by searching the recurrent communication states that have the minimum stochastic potential.

\subsection{Example}

To explain the definitions presented above, we will continue with the example of a complete network with three agents (Fig. 1). The pairs $\left(s_{1}, s_{2}\right),\left(s_{1}, s_{3}\right),\left(s_{1}, s_{4}\right)$, $\left(s_{2}, s_{5}\right),\left(s_{2}, s_{7}\right),\left(s_{3}, s_{5}\right),\left(s_{3}, s_{6}\right),\left(s_{4}, s_{6}\right),\left(s_{4}, s_{7}\right),\left(s_{5}, s_{8}\right),\left(s_{6}, s_{8}\right)$, and $\left(s_{7}, s_{8}\right)$ are adjacent states. The direction of any arrow from adjacent states $s_{i}$ to $s_{j}$ represents resistance $r\left(s_{i}, s_{j}\right)$. If the arrow starts from $s_{i}$ then $r\left(s_{i}, s_{j}\right)=0$; otherwise $r\left(s_{i}, s_{j}\right)=1$. The resistance of any path of adjacent states is equal to the sum of the resistances between adjacent states on the path. For example, for the path $\left(s_{1}, s_{2}, s_{5}, s_{8}\right), r\left(s_{1}, s_{2}, s_{5}, s_{8}\right)=r\left(s_{1}, s_{2}\right)+r\left(s_{2}, s_{5}\right)+r\left(s_{5}, s_{8}\right)=1$. To find the resistance between non-adjacent states we need to find the path with minimum resistance. For instance, there are six different paths connecting $s_{1}$ and $s_{8}$. These are $\left(s_{1}, s_{2}, s_{5}, s_{8}\right)$, $\left(s_{1}, s_{2}, s_{7}, s_{8}\right),\left(s_{1}, s_{3}, s_{5}, s_{8}\right),\left(s_{1}, s_{3}, s_{6}, s_{8}\right),\left(s_{1}, s_{4}, s_{6}, s_{8}\right)$, and $\left(s_{1}, s_{4}, s_{7}, s_{8}\right)$. The resistances of these paths all equal to 1 . One can say that $r\left(s_{1}, s_{8}\right)=1$. Note that resistance is not a symmetric binary operation, e.g. $1=r\left(s_{1}, s_{8}\right) \neq r\left(s_{8}, s_{1}\right)=2$.

In this example, for each state $s$, there exist $384\left(3 \times 2^{7}\right)$ different s-trees rooted in $s$. In Table 1 we depict three of s-trees rooted in $s_{1}$ and $s_{8}$. A directed edge $\left(s, s^{\prime}\right)$, where $s$ and $s^{\prime}$ are adjacent states, is represented by an arrow from $s$ to $s^{\prime}$. The number next to each arrow from $s$ to $s^{\prime}$ is $r\left(s, s^{\prime}\right)$. In each s-tree in (i)-(iii) [(iv)-(vi)], for any state $s^{\prime}$, there is a unique directed path leading from $s^{\prime}$ to $s_{1}\left[s_{8}\right]$.

For both $s_{1}$ and $s_{8}$, there are three types of s-trees in terms of the sum of resistance of the directed paths. The sums of resistances of $s_{1}$-trees shown in (i)-(iii) are 2, 3, and 4 , respectively. Likewise, the sums of resistances of $s_{8}$-trees shown in (iv)-(vi) are 1 , 2 , and 3 , respectively. ${ }^{12}$ That is, the stochastic potential of $s_{1}$ is 2 and the stochastic potential of $s_{8}$ is 1 .

Observe that for all states in state space $S$, there is a path of zero resistance to a recurrent communication state. So, in order to detect minimal stochastic potential recurrent communication states, as Young's Theorem (1993) suggested, we need to consider the only possible paths between recurrent communication states. One can easily notice from Fig. 1 that there are only two states $\left(s_{1}\right.$ and $\left.s_{8}\right)$ where any path has a positive resistance, i.e. these are the only recurrent communication states. In Table 2 we depict all possible trees rooted in $s_{1}$. Each of these trees is a minimal resistance $s_{1}$-tree and the sum of resistances of each tree is $2(1+1+0)$ which is the stochastic potential of $s_{1}$. Similarly, the stochastic potential of $s_{8}$ is $r\left(s_{1}, s_{8}\right)=2$. That is, we need only one tremble to reach the state where everyone adopts the new network product, whereas one more tremble is needed to return back to the state where no one uses the new network product. Therefore, $s_{8}$ is the only stochastically stable equilibrium.

\footnotetext{
12 For $s_{1}\left(s_{8}\right)$, there are 54 unique s-trees that have the sum of resistances equal to 2(1), 180 unique s-trees that have the sum of resistances equal to 3(2), and 150 unique s-trees that have the sum of resistances equal to 4(3).
} 


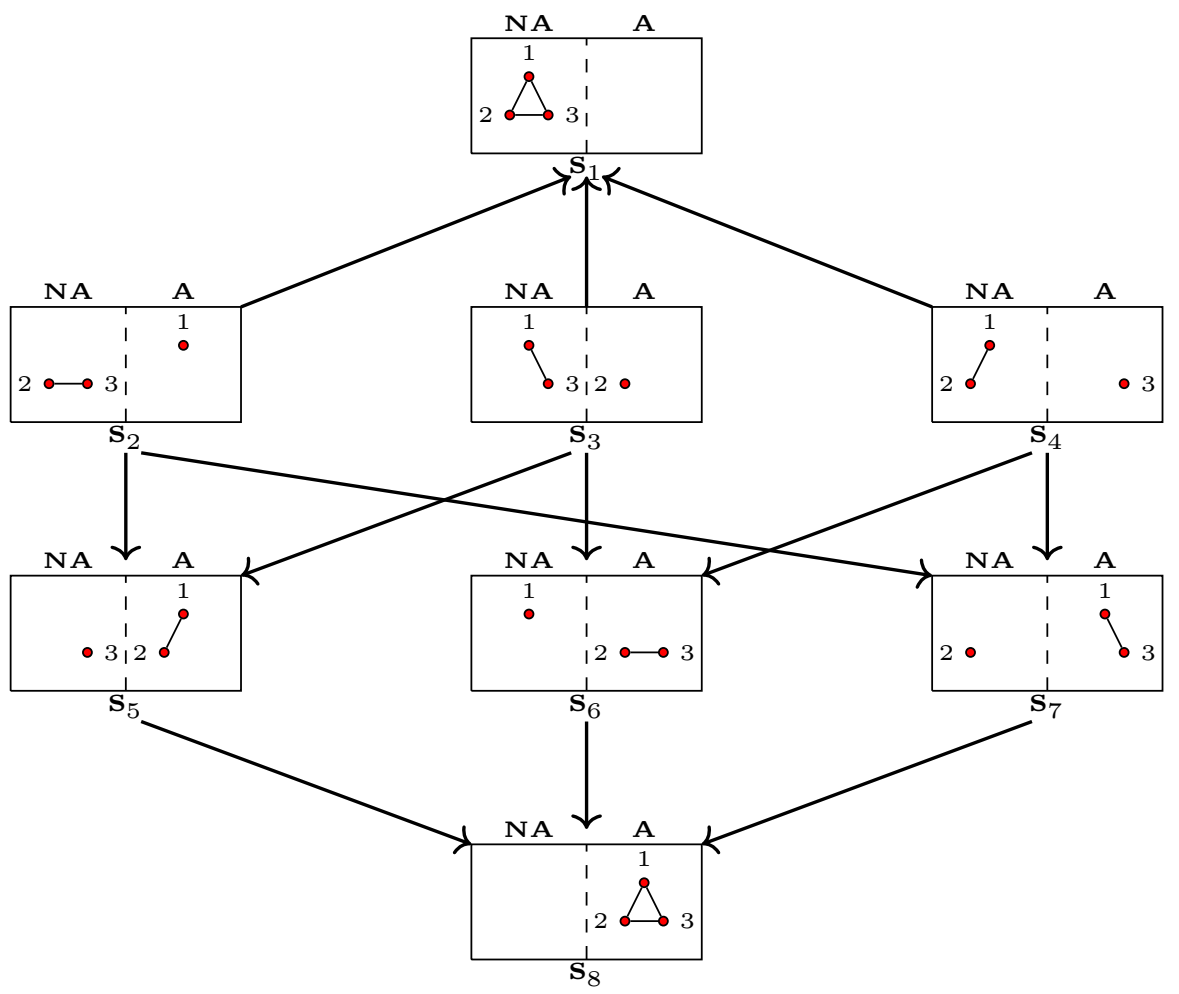

Fig. 1 The Markov chain of the complete network with three agents

Table 1 Examples of $s_{1}$-tree and $s_{8}$-tree

\begin{tabular}{|c|c|c|}
\hline 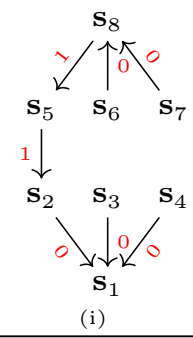 & 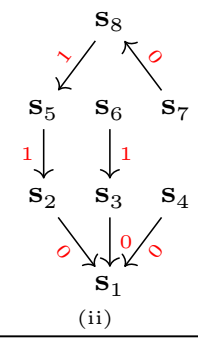 & 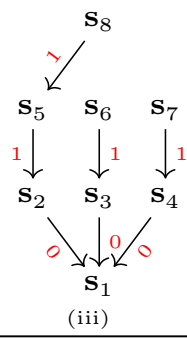 \\
\hline 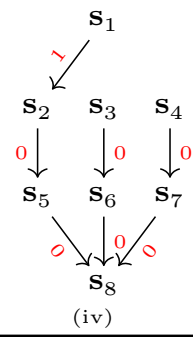 & 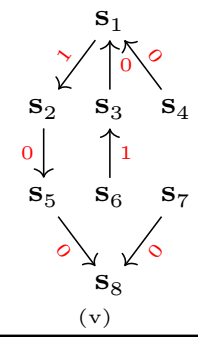 & 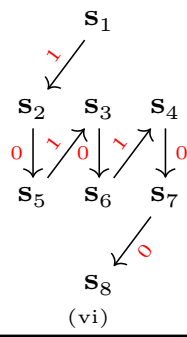 \\
\hline
\end{tabular}


Table 2 Possible trees rooted in $s_{1}$

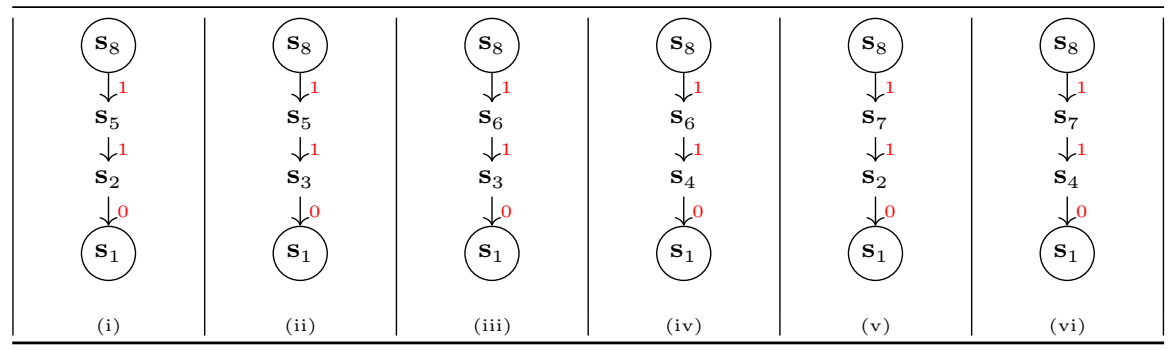

\section{Theoretical results}

\subsection{The case of symmetric network}

In the following two propositions, we characterize the stochastically stable equilibria for complete networks and cycles.

Proposition 1 For any complete network $g$, with size $n$ :

(i) If $n$ is odd, then the unique stochastically stable equilibrium is $s_{A}$,

(ii) If $n$ is even, then the two stochastically stable states in this case are $s_{N A}$ and $s_{A}$.

Proof See "Appendix".

Understanding the following example is key to understanding Proposition 1.

Example 1 (Complete network) Let $n=5$, all agents are located on a complete network. In this case, the state space $S$ contains 32 states. Two of them are $s_{N A}$ and $s_{A}$, in which none of the agents switches to a different adoption status when all of his neighbors share the same status. That is, the resistance of any path from these states to an adjacent state is positive. On the other hand, when there are two or fewer agents who adopt (three or fewer agents who do not adopt) the new network product, there is a path of zero resistance to $s_{N A}\left(s_{A}\right)$. This means that two trembles are needed to reach $s_{A}$ from $s_{N A}$, whereas three trembles are needed to return from $s_{A}$ back to $s_{N A}$. Thus, the unique stochastically stable state in this case is for all agents to adopt the new network product.

Proposition 2 For any cycle $g$ with any size $n$, the unique stochastically stable equilibrium is $s_{A}$.

Proof See "Appendix".

The intuition behind Proposition 2 is somehow different from that of Proposition 1. Note that when no one adopts the new network product, the first randomly chosen agent will adopt the new network product with probability $\varepsilon$. After his adoption of the new network product, any of the neighbors of this agent will also choose to adopt the same network product, because each has one neighbor in both adoption status 
networks. As a result, the rest of the network will voluntarily choose to adopt the new network product one by one. The choice (to adopt the new network product) spreads in the population in the form of a contagion, as in Morris (2000). However, with probability less than $\varepsilon^{2}$ the system will reach $s_{N A}$ from $s_{A}$. That is, one tremble is needed to reach $s_{A}$ from $s_{N A}$, whereas more than two trembles are needed to return from $s_{A}$ back to $s_{N A}$. Thus, the unique stochastically stable state in this case is for all agents to adopt the new network product.

\subsection{The general case}

In complete networks and cycles, all agents would act together in every equilibria of the adoption game. On the other hand, for an arbitrary network structure, the calculations to characterize the stochastically stable equilibria are demanding for even a small number of agents. However, if the set of recurrent communication states, which is a superset of stochastically stable equilibria, is known, then we can exploit the tree construction method of Freidlin and Wentzell (1998) in order to fully characterize the stochastically stable equilibria (Jackson and Watts 2002a). Therefore, we aim to characterize the set of recurrent communication states for an arbitrary network. To do so we need to introduce some definitions and a decomposition procedure for a network.

For any network $g$ a subgraph $D$ is a strong pack (weak pack) if the number of connections of agent $i$ within subgraph $D$ is greater than (greater than or equal to) the number of connections of agent $i$ outside the subgraph $D$. In a strong pack (weak pack) $D$, each agent has more than (more than or equal to) half of his links inside the pack $D$. A weak pack $D$ is a saturated pack if $g \backslash D$ is a strong pack but $D$ is not. In a saturated pack $D$, there exists an agent who has an equal number of links within and outside the pack $D$. Since $g \backslash D$ is a strong pack, no additional agent will join to the pack $D$, in some cases, however, some agents may move to $g \backslash D$ from $D$.

A network $g$ is decomposable into strong packs if $g$ is partitioned into a collection of subgraphs $\left\{D_{1}, \ldots, D_{k}\right\}$ such that each of them is a strong pack. ${ }^{13}$

We observe that for an arbitrary network, the structure of recurrent communication states depends on the following conditions.

Condition 1 There exists a saturated pack of the network $g$.

Condition $2 \mathrm{~g}$ is decomposable into strong packs.

For an arbitrary network, Proposition 3 characterizes the recurrent communication states of the adoption game. Before proceeding to the results, let us define some specific subsets of the state space $S$ :

- A state $s \in S_{1}$ if and only if there exists a saturated pack $D$ of $g$ such that every agent in $D$ adopts the new network product and every agent in $g \backslash D$ shares the same adoption status in the state $s$.

\footnotetext{
13 The decomposition of a network into strong packs may not be unique. There may exist two collections of subgraphs $\left\{D_{1}^{1}, \ldots, D_{k_{1}}^{1}\right\}$ and $\left\{D_{1}^{2}, \ldots, D_{k_{2}}^{2}\right\}$ such that each $D_{i}^{1}$ and $D_{j}^{2}$ are strong packs and both collections partition the vertex set of the network.
} 
Table 3 Decomposition into strong packs

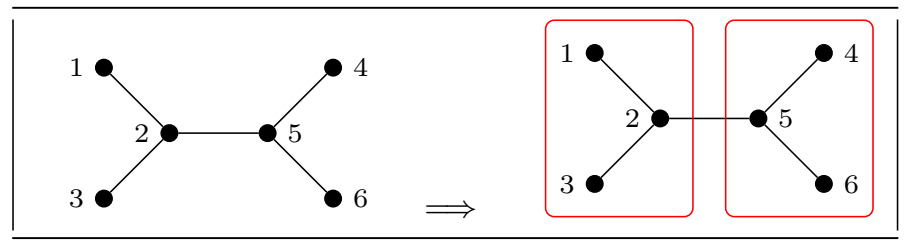

- A state $s \in S_{2}$ if and only if there exists a collection of strong packs $\left\{D_{1}, \ldots, D_{k}\right\}$ that partition $g$ such that for any $j \in\{1, \ldots, k\}$ every agent in $D_{j}$ shares the same adoption status in the state $s$.

$-S_{p}=\left\{s_{N A}, s_{A}\right\}$, where $s_{N A}$ and $s_{A}$ are the polar states.

Notice that if a network $g$ does not satisfy Condition 1 (or Condition 2), then $S_{1}$ (or $S_{2}$ ) is empty.

Proposition 3 For an arbitrary network $g$ the set of recurrent communication states is $S_{1} \cup S_{2} \cup S_{p}$.

Proof Given a network $g$ and a state $s$, let $D_{N A}$ be the subgraph of all agents not adopting the new network product and let $D_{A}$ be the subgraph of all agents adopting the new network product. Suppose that $s$ is a recurrent communication state with $D_{N A} \neq \varnothing$ and $D_{A} \neq \varnothing$. It is thus trivial from the definition that $D_{N A}$ is a strong pack and $D_{A}$ is a weak pack. If $D_{A}$ is a strong pack, $s \in S_{2}$. If $D_{A}$ is not a strong pack ( $D_{N A}$ is a saturated pack), $s \in S_{1}$. In addition, a recurrent communication state $s$ with $D_{N A}=\varnothing$ or $D_{A}=\varnothing$ belongs to $S_{p}$. This shows that a recurrent communication state $s$ belongs to $S_{1} \cup S_{2} \cup S_{p}$. The converse holds trivially and the proposition is proved.

Because the characterization of the stochastically stable equilibria depends on the fine details of the network, we are not able to characterize these equilibria for an arbitrary network. However, recurrent communication states provide us a sharp insight about what the equilibria would look like. In addition, the recurrent communication states may coincide with that of stochastically stable equilibria, as in the following example (Table 3).

Example 2 (Network $g_{1}$ ) Notice that the network $g_{1}$ satisfies Condition 2 but not Condition 1.

$D_{1}=\{(1,2),(2,3)\}$ and $D_{2}=\{(4,5),(5,6)\}$ are the only strong packs that partition $g_{1}$, i.e. $g_{1}$ is decomposable into strong packs. So according to Proposition 3 , the recurrent communication states of the adoption process are: 
Table 4 All possible $s_{N A}$-trees

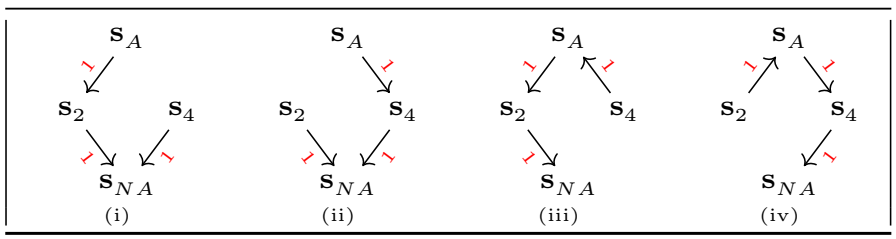

In Table 4 , we depict all possible $s_{N A}$-trees. ${ }^{14}$ Note that each $s_{N A}$-tree has resistance equal to 3 , i.e. the stochastic potential of $s_{N A}$ is 3 . One can easily calculate that the stochastic potentials of the other recurrent communication states are also 3 . Therefore, in this case every recurrent communication state is a stochastically stable equilibrium.

\section{Simulation model}

As we mentioned earlier, because the characterization of the stochastically stable equilibria depends on the fine details of the network, we are not able to characterize stochastically stable equilibria for an arbitrary network. In this section, we use a generalization of the Morris (2000) Contagion model to analyze diffusion of network products in small-world networks. Also, with this model, we try to explain the chasm between early adopters and early majority.

\subsection{The network}

There is an exogenously given undirected communication network that connects a finite set of agents $N=\{1, \ldots n\}$. For any network $g$ and for any $i, j \in N,(i, j) \in g$ means that agent $i$ talks to agent $j$. In addition, $N_{i}(g)$ will denote the set of agents linked with agent $i$. Formally:

$$
N_{i}(g)=\{j \in N \text { such that }(i, j) \in g\} .
$$

\subsection{The game}

Let's assume that the market offers a new network product. Each agent has two possible adoption statuses: adopting (1) or non-adopting (0). We assume that each agent chooses a constant action for all his neighbors. Let $u_{i}\left(s, s^{\prime}\right)$ be the utility of an agent when he chooses status $s$ and his neighbor chooses status $s^{\prime}$. This yields the following payoff matrix:

\footnotetext{
14 An s-tree for a state is defined in Sect. 3.3.
} 


\begin{tabular}{llr}
\hline & 0 & 1 \\
\hline 0 & $u_{i}(0,0), u_{j}(0,0)$ & $u_{i}(0,1), u_{j}(1,0)$ \\
1 & $u_{i}(1,0), u_{j}(0,1)$ & $u_{i}(1,1), u_{j}(1,1)$ \\
\hline
\end{tabular}

As in Morris (2000), we assume that this game has two strict Nash equilibria. So, we need $u_{i}(0,0)>u_{i}(1,0)$ and $u_{i}(1,1)>u_{i}(0,1)$ for any $i \in N$. Notice that if agent $i$ assigns probability at least $p_{i}$ to the other agent adopting a new network product, then adopting the same network product is the best response for him. The associated $p_{i}$ is calculated as:

$$
p_{i}=\frac{u_{i}(0,0)-u_{i}(1,0)}{u_{i}(0,0)-u_{i}(1,0)+u_{i}(1,1)-u_{i}(0,1)} .
$$

Similar to Morris (2000), we can parameterize the payoffs as such:

\begin{tabular}{lll}
\hline & 0 & 1 \\
\hline 0 & $p_{i}, p_{j}$ & 0,0 \\
1 & 0,0 & $1-p_{i}, 1-p_{j}$ \\
\hline
\end{tabular}

We assume that each agent's best response is to choose an action which maximizes the sum of his utilities arising from the interactions with his neighbors. Thus, for any network $g$, if

$$
\sum_{j \in N_{i}(g)} u_{i}\left(1, s_{j}\right) \geq \sum_{j \in N_{i}(g)} u_{i}\left(0, s_{j}\right),
$$

then adopting a new network product is the best response to the collection of $\left\{s_{j}\right\}_{j \in N_{i}(g)}$ such that $s_{j} \in\{0,1\}$. As a result, adopting a new network product is the best response for agent $i$ if at least proportion $p_{i}$ of his neighbors adopted the same network product. Observe that in the theoretical model, for each agent $i$, the threshold proportion equals to $1 / 2$, i.e. $p_{i}=1 / 2$.

\subsection{The simulation procedure}

First, we simulate the network as a Watts and Strogatz (1998) small-world network. All the simulations are made starting from a regular network of 1000 agents with a degree of 10 each. As $\beta$ varies, some initial links are deleted and some new links are formed, but on average each agent has 10 links in each case. Secondly, each agent is assigned a random probability $p_{i}$ for use as a threshold value in adoption decisions. These probabilities are calibrated using the parameters in Choi et al. (2010).

We confirm Choi et al.'s (2010) results if we start with a perfect 1-lattice in which each agent has precisely 20 links each. This indicates that innovators have less impact on their friends' adoption decision than what they have in our model. The low observed 


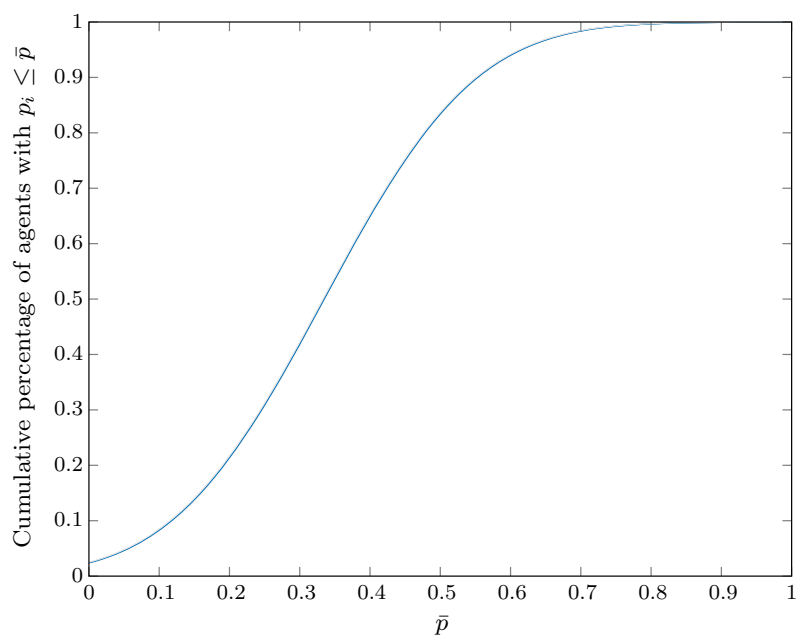

Fig. 2 Cumulative distribution of the assigned threshold probabilities

effect of innovators may yield a larger probability of under-adoption of the network product.

Figure 2 gives the cumulative distribution of the threshold probabilities assigned to agents. ${ }^{15}$ Notice that about $2.5 \%$ of the total population (as suggested by Rogers 2010) are chosen to be innovators (i.e. for these agents $p_{i}=0$ ). Specifically, there are initially some agents who voluntarily adopt a new network product without any network effects.

At a given discrete time $t$, any non-adopting agent who is connected to any of the previous adopters makes a decision. Agent $i$ adopts a new network product if at least proportion $p_{i}$ of his neighbors adopted the same network product. The process repeats until either every agent has adopted the new network product or the system reaches the steady state, i.e. no new adopter arises.

There are two main differences between the theoretical and simulation models. First, in the theoretical model, a randomly chosen agent makes the decision in each period. This agent can also belong to an adopting network. In the simulation model, in order to reduce the process time, we only choose agents from non-adopting network. Additionally, in each period $t$, every agent in a non-adopting network makes a decision considering the network structures in the period $t-1$.

Second, in the theoretical model, every agent is initially in the non-adopting network, i.e. no one voluntarily adopts the new network product. To initiate the process we need a tremble. However, in the simulation model, there are agents_- "innovators"who have $p_{i}=0$, meaning that at period 1 these agents will voluntarily adopt the new network product. So, the innovators initiate the adoption process in the simulation model.

15 All data points in Figs. 2, 3, 4 and 5 are obtained by taking averages of 10,000 simulations. The shaded area around each line represents the $95 \%$ confidence interval, but due to the large number of simulations these confidence intervals are generally narrow. 


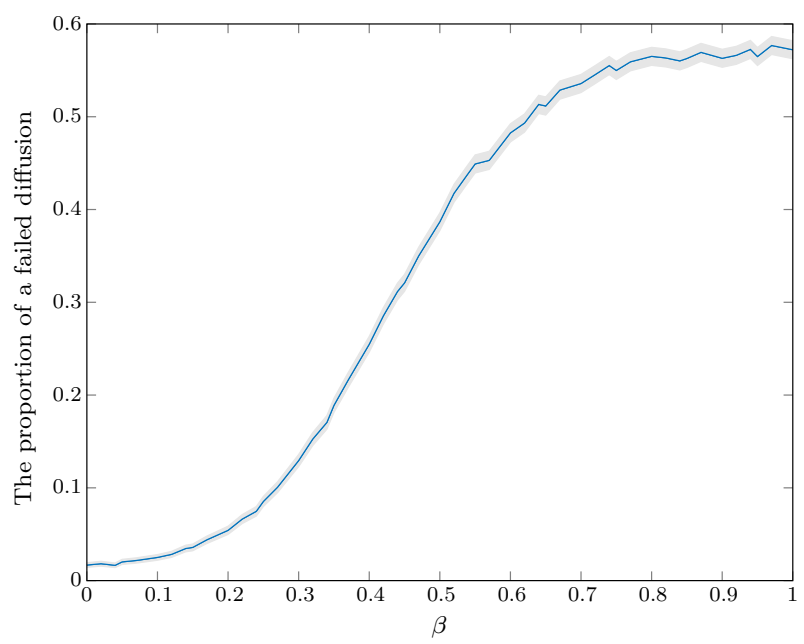

Fig. 3 Proportion of the failed contagion depending on $\beta$

\section{Simulation results}

In our study, the process is said to have failed if it stops before $16 \%$ of total agents adopt the new network product. That is, if no agent from the early majority adopts the new network product, this will imply a failed contagion. Figure 3 illustrates that as $\beta$ gets bigger, the likelihood of a contagion diffusion converges to about 45 percent.

Figure $4 \mathrm{a}$ indicates that when $\beta$ is close to 0 , the probability distribution of the number of adopters at a steady state is almost normal. As $\beta$ is close to 0.15 the type of the distribution skews to the left. When $\beta \geq 0.15$, the number of adopters at a steady state has a bimodal distribution (Fig. 4b). That is, either the diffusion is failed or there is a full contagion.

Figure $4 \mathrm{~b}$ is also consistent with Moore's (2002) "notion of chasm". Notice that when $\beta \geq 0.15$, if the process reaches a state in which at least $16 \%$ of total agents adopted the new network product, then this will result an almost full contagion. Strictly speaking, there is a chasm between early adopters and early majority, and if the process crosses this chasm, this brings a full diffusion with a higher probability. Figure 3 also suggests that the larger the $\beta$, the more difficult it is to cross the chasm.

Additionally, in Fig. 5 we present the relation between network structure $(\beta)$ and the speed of contagion. ${ }^{16}$ When $\beta=0$, the process reaches a steady state when time equals 40 . Even though the average time taken to reach a steady state increases as $\beta$ is close to 0.1 , it drops dramatically between 0.1 and 0.2 . The average time taken to reach a steady state is shortest when $\beta \geq 0.7$. Particularly, random links provide a rapid contagion, but more random links do not necessarily imply a more rapid contagion.

\footnotetext{
16 For Fig. 5, we only considered the cases in which at least $50 \%$ of the total agents adopt the new network product. 

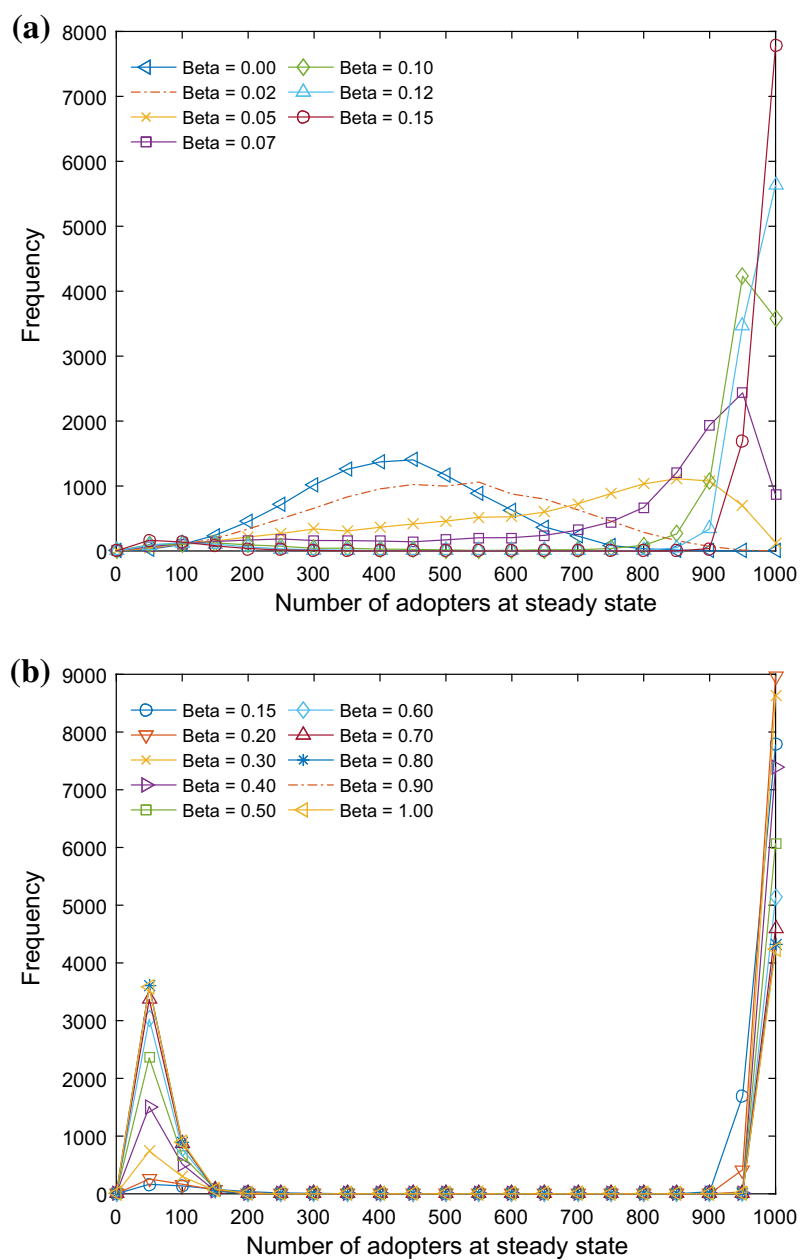

Fig. 4 Frequency distributions of the contagion depending on $\beta$

\section{Concluding remarks}

In this paper, we have adopted a generic model for 0-1 type decisions on networks in which agents consider only close friends' decisions. One of our main contributions is to show how the contagion process and the network structure of the social contacts jointly evolve. We have characterized the stochastically stable equilibria for complete networks and cycles. We have proposed that agents act together if the network structure is symmetric. For arbitrary network structures, we have provided a new method to characterize the recurrent communication states that are candidates for being a stochastically stable equilibrium.

In the simulation model, we have studied the contagion process of a network product in small-world networks. Also, this type of network allows us to analyze two extreme 


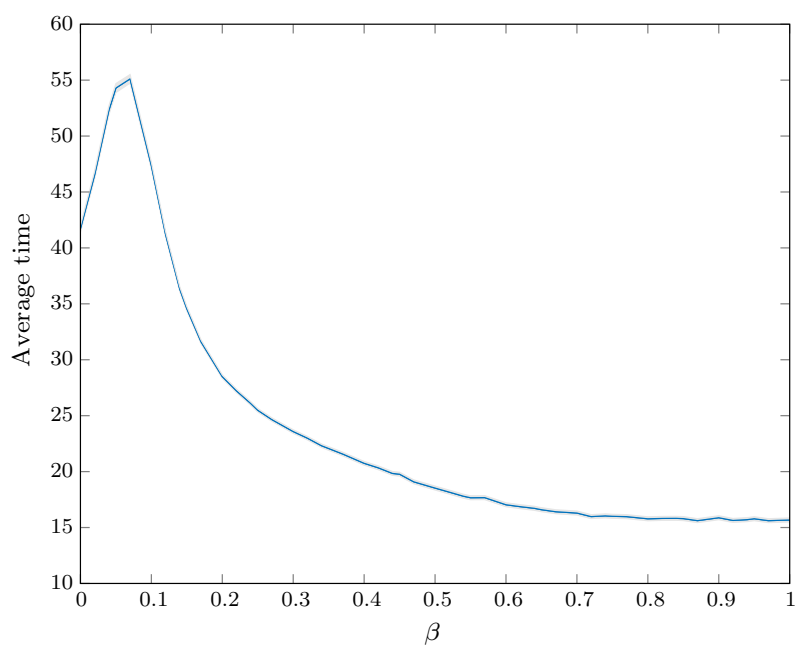

Fig. 5 Average time to reach a steady state depending on $\beta$

cases: highly cliquish networks and random networks. Our numerical analysis shows that failure of a new network product is less likely in a highly cliquish network. In addition, the average time taken to reach a steady state is shortest when $\beta$ is greater than or equal to 0.7 . Namely, the contagion process reaches a steady state faster in random networks than in highly cliquish networks. Our simulation model results show that a new network product succeeds in a random network with probability 0.45 , which is higher than what was found in Choi et al. (2010) $(\sim 0.1)$. This difference is mainly driven by the average number of links the agents have in the simulation procedure. ${ }^{17}$

Choi et al. (2010) and Granovetter (1973) also point out that agents get more useful job information from random connections, i.e. random networks are more beneficial for the contagion of job information. It seems that our model exhibits results that are closer what Granovetter (1973) found than Choi et al.'s (2010) model.

As previously mentioned, our simulation model indicates that a new network product succeeds in a random network with probability 0.45 . Interestingly, this result rationalizes the well-known quote "Half the money I spend on advertising is wasted; the trouble is I don't know which half”, by John Wanamaker.

Using our simulation model, we could explain the chasm between early adopters and early majority. We have seen that full diffusion is more likely if the process crosses this chasm.

This simulation model confirms our theoretical results. Notice that a network that satisfies Condition 1 or Condition 2 is most likely a cliquish network. If none of these conditions holds, the only candidates for equilibrium are the polar states. Similarly, in the simulation model, when $\beta \geq 0.15$, the number of adopters at a steady state has a bimodal distribution.

It turns out that marketers should focus on one particular group of customers at a time. Also, working with mixed marketing strategies (creating positive word of

\footnotetext{
17 We discussed this issue in detail in Sect. 5.3.
} 
mouth and moderate advertising campaigns) will result in a higher probability of full contagion of network products and faster contagion rates. ${ }^{18}$

Acknowledgements I thank the editor, the associate editor, and two anonymous reviewers for their thoughtful and constructive comments. I am also indebted to Semih Akçomak, Pelin Akyol, Rahmi İlkılıç, Ayşe Özgür Pehlivan, and Kemal Yıldız for helpful conversations and discussions. The views expressed here are my own and do not necessarily reflect those of the Presidency of the Republic of Turkey Presidency of Strategy and Budget. Any errors are my own.

\section{Appendix}

\section{A Proofs}

Proof of Proposition 1 Claim For any state $s$, there is a path of zero resistance leading to either $s_{N A}=(0,0, \ldots, 0)$ or $s_{A}=(1,1, \ldots, 1)$.

Proof of Claim For a given state $s$, let $n_{0}$ denote the number of agents in the nonadopting network and $n_{1}$ denote the number of agents in the adopting network.

Case $1 n_{0}>n_{1}$

Any agent in the adopting network will voluntarily switch to the non-adopting network at the time of the adoption decision, since each of them has more than half of their connections in the non-adopting network. This will continue until the last agent in the adopting network switches to the non-adopting network. Thus, there is a path of zero resistance leading to $s_{N A}$.

Case $2 n_{0} \leq n_{1}$

Any agent in the non-adopting network will adopt the new network product, since each of them has at least half of their connections in the adopting network. This adoption process will continue until everyone adopts the new network product. Hence, there is a path of zero resistance leading to $s_{A}$.

Note also that any path from $s_{N A}$ or $s_{A}$ to any adjacent state has a positive resistance. Hence the only recurrent communication states are $s_{N A}$ and $s_{A}$. Given Young's Theorem, we need to find which of these states has the smallest stochastic potential.

We will find the minimum resistance over paths from $s_{N A}$ to $s_{A}$ and will compare the minimum resistance over paths from $s_{A}$ to $s_{N A}$. If everyone is in the non-adopting network, then it takes $(n-1) / 2$ trembles if $\mathrm{n}$ is odd and $n / 2$ trembles if $n$ is even to get everyone adopt the new network product. If everyone is in the adopting network, then for everyone to not adopt the new network product, it takes $(n+1) / 2$ trembles if $n$ is odd and it takes $n / 2$ trembles if $\mathrm{n}$ is even.

Thus, if $n$ is odd $r\left(s_{N A}, s_{A}\right)<r\left(s_{A}, s_{N A}\right)$, i.e. $s_{A}$ is the unique stochastically stable equilibrium. If $n$ is even $r\left(s_{N A}, s_{A}\right)=r\left(s_{A}, s_{N A}\right)$, i.e. $s_{N A}$ is also a stochastically stable.

Proof of Proposition 2 Claim For any state $s$, there is a path of zero resistance leading to either $s_{N A}$ or $s_{A}$.

$18 \mathrm{Hu}$ and Zu's (2017) finding, "mass media can change agents' choices not only directly by global broadcasting but also indirectly by peer influence among agents within social networks" also supports our conclusion. 
Proof of Claim For a given state $s$ there are three cases:

Case $1 s=s_{N A}$

Case $2 s=s_{A}$

Cases 1-2 are trivial cases. In each state, no one voluntarily leaves, i.e. a randomly chosen agent continues to stay in that state. Thus, there is a path of zero resistance leading to either $s_{N A}$ if $s=s_{N A}$ or $s_{A}$ if $s=s_{A}$.

Case 3 There is at least one agent in both status networks.

The connectedness assumption ensures that there exists an agent in the non-adopting network who has at least one neighbor in the adopting network. Then, this agent will adopt the new network product when he makes an adoption decision. If the updated state is not $s_{A}$, again by the connectedness assumption there exists an agent who has at least one neighbor in the adopting network. At the time of his adoption decision, he will also voluntarily adopt the new network product. This contagion process will continue until the updated state is $s_{A}$. Thus, there is a path of zero resistance leading to $s_{A}$.

Note also that any path from $s_{N A}$ or $s_{A}$ to any adjacent state has a positive resistance. Hence the only recurrent communication states are $s_{N A}$ and $s_{A}$. Given Young's Theorem, we need to find which of these states has the smallest stochastic potential. If everyone is in the non-adopting network, no agent will voluntarily adopt the new network product. If an agent adopts the new network product due to an $\varepsilon$-error, then we reach a state that has a path of zero resistance leading to $s_{A}$. So, $r\left(s_{N A}, s_{A}\right)=1$. If everyone is in the adopting network and an agent switches to the non-adopting status due to an $\varepsilon$-error, then we reach a state where each agent has at least one neighbor in the adopting network. It takes at least more than two trembles to get all agents to switch to the non-adopting network. So, $r\left(s_{A}, s_{N A}\right)>2$.

Thus the unique stochastically stable equilibrium is $s_{A}$.

\section{References}

Babutsidze Z, Cowan R (2014) Showing or telling? Local interaction and organization of behavior. J Econ Interact Coord 9(2):151-181

Bargigli L, Tedeschi G (2013) Major trends in agent-based economics. J Econ Interact Coord 8:211-217

Bass FM (1969) A new product growth for model consumer durables. Manag Sci 15(5):215-227

Boccaletti S, Latora V, Moreno Y, Chavez M, Hwang DU (2006) Complex networks: structure and dynamics. Phys Rep 424(4-5):175-308

Chakravartula S (2014) Complex networks: structure and dynamics. University of Massachusetts Boston, Boston

Choi H, Kim SH, Lee J (2010) Role of network structure and network effects in diffusion of innovations. Ind Mark Manag 39(1):170-177

Dunbar RI (1998) The social brain hypothesis. Evol Anthropol Issues News Rev Issues News Rev 6(5):178190

Dunbar RI, Arnaboldi V, Conti M, Passarella A (2015) The structure of online social networks mirrors those in the offline world. Soc Netw 43:39-47

Erdos P, Rényi A (1960) On the evolution of random graphs. Publ Math Inst Hung Acad Sci 5(1):17-60

Foster D, Young P (1990) Stochastic evolutionary game dynamics. Theor Popul Biol 38(2):219

Freidlin MI, Wentzell AD (2012) Random perturbations of dynamical systems. Translated from the 1979 Russian original by Joseph Szücs. Third edition. Grundlehren der Mathematischen Wissenschaften [Fundamental Principles of Mathematical Sciences], vol. 260. Springer, Heidelberg. https://doi.org/ 10.1007/978-3-642-25847-3 
Fuchs B, Sornette D, Thurner S (2014) Fractal multi-level organisation of human groups in a virtual world. Sci Rep 4:6526

Gilchrist DS, Sands EG (2016) Something to talk about: social spillovers in movie consumption. J Polit Econ 124(5):1339-1382

Granovetter M (1973) The strength of weak ties. Am J Sociol 78(6):1360-1380

Granovetter M (1978) Threshold models of collective behavior. Am J Sociol 83(6):1420-1443

Granovetter M (1995) Getting a job: a study of contacts and careers. University of Chicago Press, Chicago

Hill RA, Dunbar RI (2003) Social network size in humans. Hum Nat 14(1):53-72

Hu H, Zhu JJ (2017) Social networks, mass media and public opinions. J Econ Interact Coord 12(2):393-411

Ioannides YM, Topa G (2010) Neighborhood effects: accomplishments and looking beyond them. J Reg Sci 50(1):343-362

Jackson MO, Watts A (2002a) The evolution of social and economic networks. J Econ Theory 106(2):265295

Jackson MO, Watts A (2002b) On the formation of interaction networks in social coordination games. Games Econ Behav 41(2):265-291

Kiesling E, Günther M, Stummer C, Wakolbinger LM (2012) Agent-based simulation of innovation diffusion: a review. Centr Eur J Oper Res 20(2):183-230

Lelarge M (2012) Diffusion and cascading behavior in random networks. Games Econ Behav 75(2):752-775

Lehmann DR, Parker JR (2017) Disadoption. AMS Rev 7(1-2):36-51

Mac Carron P, Kaski K, Dunbar R (2016) Calling Dunbar's numbers. Soc Netw 47:151-155

McIntyre DP, Chintakananda A (2014) Competing in network markets: can the winner take all? Bus Horiz 57(1):117-125

Miller J, Muir D (2005) The business of brands. Wiley, New York

Moore GA (2002) Crossing the chasm. Harpercollins, New York

Moore C, Newman ME (2000) Epidemics and percolation in small-world networks. Phys Rev E 61(5):5678

Morris S (2000) Contagion. Rev Econ Stud 67(1):57-78

Newman ME, Watts DJ (1999) Scaling and percolation in the small-world network model. Phys Rev E 60(6):7332

Pegoretti G, Rentocchini F, Marzetti GV (2012) An agent-based model of innovation diffusion: network structure and coexistence under different information regimes. J Econ Interact Coord 7(2):145-165

Peres R (2014) The impact of network characteristics on the diffusion of innovations. Phys A Stat Mech Appl 402:330-343

Richerson PJ, Boyd R (1998) The evolution of human ultrasociality. In: Indoctrinability, ideology, and warfare: evolutionary perspectives, pp 71-95

Rogers EM (2010) Diffusion of innovations. Simon and Schuster, New York

Sacerdote B (2001) Peer effects with random assignment: results for Dartmouth roommates. Q J Econ 116(2):681-704

Saramäki J, Leicht EA, López E, Roberts SG, Reed-Tsochas F, Dunbar RI (2014) Persistence of social signatures in human communication. Proc Natl Acad Sci 111(3):942-947

Sutcliffe A, Dunbar R, Binder J, Arrow H (2012) Relationships and the social brain: integrating psychological and evolutionary perspectives. Br J Psychol 103(2):149-168

Tauber EM (1972) Why do people shop? J Mark 36:46-49

Watts DJ, Strogatz SH (1998) Collective dynamics of 'small-world' networks. Nature 393(6684):440-442

Xiong H, Payne D, Kinsella S (2016) Peer effects in the diffusion of innovations: theory and simulation. J Behav Exp Econ 63:1-13

Young HP (1993) The evolution of conventions. Econ J Econ Soc 61:57-84

Publisher's Note Springer Nature remains neutral with regard to jurisdictional claims in published maps and institutional affiliations. 